\title{
Uncertain Linguistic Power Geometric Operators and Their Use in Multiattribute Group Decision Making
}

\author{
Huimin Zhang \\ School of Management, Henan University of Technology, Zhengzhou 450001, China \\ Correspondence should be addressed to Huimin Zhang; zhm76@126.com
}

Received 16 August 2014; Revised 2 December 2014; Accepted 23 December 2014

Academic Editor: Cheng C. Wang

Copyright (C) 2015 Huimin Zhang. This is an open access article distributed under the Creative Commons Attribution License, which permits unrestricted use, distribution, and reproduction in any medium, provided the original work is properly cited.

Based on the extended triangular norm, several new operational laws for linguistic variables and uncertain linguistic variables (ULVs) are defined. To avoid the limitations of existing linguistic aggregation operators, a series of extended uncertain linguistic (UL) geometric aggregation operators are proposed on the basis of the extended triangular norm. In addition, a multiattribute group decision making (MAGDM) method dealing with UL information is developed based on the extended UL geometric aggregation operators. Finally, an example is presented to show the efficiency of the developed approach in solving MAGDM problems.

\section{Introduction}

Due to the increasing complexity of the decision problem and our limited ability, in the multiattribute group decision making (MAGDM) process, decision makers sometimes have difficulty in providing their opinions with crisp numbers. Alternatively, by means of linguistic information, they think they can express their opinions more naturally and straightforward. The studies on decision making with linguistic information have been widely performed and many methods have been developed to solve the decision making problem with linguistic information [1-12].

Sometimes, decision makers may feel that they cannot fully or accurately express their opinions with only one linguistic term. Xu [13] proposed the concept of ULV which can be regarded as one linguistic interval and is convenient for decision makers to better express their opinions. In view of the key role that aggregation operators always play in the decision making process, many UL aggregation operators have been put forward. For instance, Xu [13] introduced the UL ordered weighted averaging (ULOWA) operator and the UL hybrid aggregation (ULHA) operator. Xu [14] developed induced uncertain linguistic OWA (IULOWA) operators, where the second components are ULVs. Xu [15] defined the uncertain multiplicative linguistic preference relation and proposed several UL geometric operators. Wei [16] proposed an UL hybrid geometric mean (ULHGM) operator. Peng et al. [17] proposed an uncertain pure linguistic hybrid harmonic averaging (UPLHHA) operator. Based on the extended triangular conorm, Lan et al. [18] defined some new operational laws for linguistic variables and put forward several extended UL aggregation operators. Motivated by Bonferroni mean, Wei et al. [19] proposed several UL Bonferroni aggregation operators. Peng et al. proposed [20] some multigranular UL prioritized aggregation operators based on the prioritized aggregation operator.

Yager [21] introduced the power-average (PA) operator and the power OWA (POWA) operator which allow arguments to support each other in the aggregation process. On this basis, Xu and Yager [22] developed the power geometric (PG) operator, weighted PG operator, and POWG operator. Due to special properties of power aggregation operators, many linguistic power aggregation operators have been proposed. Zhou and Chen [23] proposed the generalized linguistic PA operator, the weighted generalized linguistic PA operator, and the generalized linguistic POWA operator. $\mathrm{Xu}$ and Wang [24] developed 2-tuple linguistic PA operator, 2tuple linguistic weighted PA operator, and 2-tuple linguistic POWA operator. Xu et al. [25] defined the linguistic PA operator, the linguistic weighted PA operator, the linguistic POWA operator, the uncertain linguistic PA operator, and the uncertain linguistic POWA operator. 
However, most of the abovementioned linguistic and UL aggregation operators are developed on the basis of the basic operational laws, by which the computing results derived sometimes may be beyond the discourse domain of the original linguistic variable and lead to a question on how to define the semantics for it. In addition, up to now, there is no PG operator developed for dealing with UL decision making problems. To overcome such issues, motivated by Lan et al. [18], we introduce the extended triangular norm and propose several uncertain linguistic PG operators based on the extended triangular norm. This paper is structured as below. Section 2 briefly introduces the basic knowledge that will be used in the following sections. Section 3 proposes several UL geometric operators based on extended triangular norm. In Section 4, a MAGDM method dealing with UL information is developed. In Section 5, an example is presented to show the efficiency of the developed approach in solving MAGDM problems. Section 6 gives the conclusions.

\section{Preliminaries}

Suppose that $S=\left\{s_{i} \mid i=1,2, \ldots, t\right\}$ is a linguistic term set (LTS), where the odd $t$ expresses the cardinality and $s_{i}$ denotes a possible linguistic value. For instance, a LTS with seven terms is expressed as below.

$S=\left\{s_{1}=\right.$ none, $s_{2}=$ very low, $s_{3}=$ low, $s_{4}=$ medium, $s_{5}=$ high, $s_{6}=$ very high, $s_{7}=$ perfect $\}$ [6].

The LTS has the properties as below [6]:

(1) $s_{j} \geq s_{k}$, if and only if $j \geq k$,

(2) negation operator: $\operatorname{Neg}\left(s_{j}\right)=s_{k}$ such that $k=t+1-j$.

$\mathrm{Xu}$ [26] proposed a continuous LTS $\bar{S}=\left\{s_{\alpha} \mid s_{1} \leq s_{\alpha} \leq\right.$ $\left.s_{t}, \alpha \in[1, t]\right\}$, where $s_{\alpha}$ is an original linguistic term if $s_{\alpha} \in S$; otherwise, $s_{\alpha}$ is a virtual linguistic term.

Suppose that $s_{\alpha}, s_{\beta} \in \bar{S}$, and $\mu, \mu_{1}, \mu_{2} \in[0,1] ; \mathrm{Xu}$ [26] gave the following operational laws:

$$
\begin{gathered}
\left(s_{\alpha}\right)^{\mu}=s_{\alpha^{\mu}}, \\
\left(s_{\alpha}\right)^{\mu_{1}} \otimes\left(s_{\alpha}\right)^{\mu_{2}}=\left(s_{\alpha}\right)^{\mu_{1}+\mu_{2}}, \\
\left(s_{\alpha} \otimes s_{\beta}\right)^{\mu}=\left(s_{\alpha}\right)^{\mu} \otimes\left(s_{\beta}\right)^{\mu}, \\
s_{\alpha} \otimes s_{\beta}=s_{\beta} \otimes s_{\alpha}=s_{\alpha \beta} .
\end{gathered}
$$

Besides, Xu [13] defined the ULV with the expression $\widetilde{s}=$ $\left[s_{\alpha}, s_{\beta}\right]$, where $s_{\alpha}, s_{\beta} \in \bar{S}, s_{\beta}$ and $s_{\alpha}$ denote the upper and lower bounds, respectively. In particular, the ULV degenerates to one linguistic variable if $\alpha=\beta$. To rank ULVs, Xu [15] gave the concept of possibility degree.

Definition 1. Let $\widetilde{s}_{1}=\left[s_{\alpha_{1}}, s_{\beta_{1}}\right]$ and $\widetilde{s}_{2}=\left[s_{\alpha_{2}}, s_{\beta_{2}}\right]$ be two ULVs, and let len $\left(\widetilde{s}_{1}\right)=\beta_{1}-\alpha_{1}$ and $\operatorname{len}\left(\widetilde{s}_{2}\right)=\beta_{2}-\alpha_{2}$; the possibility degree of $\widetilde{s}_{1} \geq \widetilde{s}_{2}$ is defined as

$$
\begin{aligned}
p\left(\widetilde{s}_{1} \geq \widetilde{s}_{2}\right) & \\
= & \frac{\max \left\{0, \operatorname{len}\left(\widetilde{s}_{1}\right)+\operatorname{len}\left(\widetilde{s}_{2}\right)-\max \left(\beta_{2}-\alpha_{1}, 0\right)\right\}}{\operatorname{len}\left(\widetilde{s}_{1}\right)+\operatorname{len}\left(\widetilde{s}_{2}\right)} .
\end{aligned}
$$

If $p\left(\widetilde{s}_{1} \geq \widetilde{s}_{2}\right) \geq 0.5$, the order between $\widetilde{s}_{1}$ and $\widetilde{s}_{2}$ is denoted by $\widetilde{s}_{1}>\widetilde{s}_{2}$.

Obviously, the possibility degree has the following properties:

(1) $0 \leq p\left(\widetilde{s}_{1} \geq \widetilde{s}_{2}\right) \leq 1,0 \leq p\left(\widetilde{s}_{2} \geq \widetilde{s}_{1}\right) \leq 1$;

(2) $p\left(\widetilde{s}_{1} \geq \widetilde{s}_{2}\right)+p\left(\widetilde{s}_{2} \geq \widetilde{s}_{1}\right)=1$. In particular, $p\left(\widetilde{s}_{1} \geq \widetilde{s}_{1}\right)=$ $p\left(\widetilde{s}_{2} \geq \widetilde{s}_{2}\right)=0.5$.

If a series of ULVs $\widetilde{s}_{i}=\left[s_{\alpha_{i}}, s_{\beta_{i}}\right](i=1,2, \ldots, m)$ should be ranked, a likelihood matrix is constructed as below:

$$
P=\left(p_{i j}\right)_{m \times m}=\left[\begin{array}{cccc}
p_{11} & p_{12} & \cdots & p_{1 m} \\
p_{21} & p_{22} & \cdots & p_{2 m} \\
\vdots & \vdots & \vdots & \vdots \\
p_{m 1} & p_{m 2} & \cdots & p_{m m}
\end{array}\right]
$$

where $p_{i j}=p\left(\widetilde{s}_{i} \geq \widetilde{s}_{j}\right)$.

Thus, the order of the ULVs is obtained in accordance with the values of $p_{i}(i=1,2, \ldots, m)$ [27]:

$$
p_{i}=\frac{\sum_{j=1}^{m} p_{i j}+m / 2-1}{m(m-1)} .
$$

Definition 2 (see $[28,29])$. A $T$-norm is a mapping $T:[0,1] \times$ $[0,1] \rightarrow[0,1]$ satisfying, for all $a, b, c, d \in[0,1]$,

(1) (boundary condition) $T(a, 1)=a$;

(2) (commutativity) $T(a, b)=T(b, a)$;

(3) (associativity) $T(a, T(b, c))=T(T(a, b), c)$;

(4) (monotonicity) $T(a, b) \leq T(c, d)$ whenever $a \leq c, b \leq$ d.

Motivated by Lan et al. [18], in what follows, we extend the $T$-norm to interval $[1, t](t>1)$.

Definition 3. An extended $T^{*}$-norm is a mapping $T^{*}:[1, t] \times$ $[1, t] \rightarrow[1, t]$ satisfying, for all $a, b, c, d \in[1, t]$,

(1) (boundary condition) $T^{*}(a, t)=a$;

(2) (commutativity) $T^{*}(a, b)=T^{*}(b, a)$;

(3) (associativity) $T^{*}\left(a, T^{*}(b, c)\right)=T^{*}\left(T^{*}(a, b), c\right)$;

(4) (monotonicity) $T^{*}(a, b) \leq T^{*}(c, d)$ whenever $a \leq c$, $b \leq d$.

Let $f:[1, t] \rightarrow[1,+\infty)$ be a strictly monotone decreasing and continuous function with $f(1)=+\infty$ and $f(t)=1$.

Lan et al. [18] proposed an interactive method to build the function of $f$; that is, given the utility values of $f(1), f(2), \ldots, f(t)$ by decision makers, the nonlinear curvefit method is performed to obtain the approximate function of $f(x)$. For reason of simplicity, unless otherwise stated, we suppose $f(x)=(t-1) /(x-1)$ in the following text. Obviously, $f(x)=(t-1) /(x-1)$ has the properties that $f(1)=+\infty$ and $f(t)=1$. Furthermore, we can easily derive the inverse function of $f(x)$; that is, $f^{-1}(x)=(x+t-1) / x$. 
Definition 4. Suppose that $T_{0}:[1, t] \times[1, t] \rightarrow[1, t]$ is a binary operator; for any $a, b \in[1, t], T_{0}$ is defined as

$$
T_{0}(a, b)=f^{-1}(f(a) * f(b)),
$$

where $f^{-1}$ is the inverse function of $f$.

Theorem 5. $T_{0}(a, b)$ is an extended $T^{*}$-norm in $[1, t]$.

Proof. Let $a, b, c, d \in[1, t]$.

(1) Since $f(t)=1$, we have $T_{0}(a, t)=f^{-1}(f(a) * f(t))=$ $f^{-1}(f(a))=a$.

(2) Consider $T_{0}(a, b)=f^{-1}(f(a) * f(b))=f^{-1}(f(b) *$ $f(a))=T_{0}(b, a)$.

(3) Consider $T_{0}\left(a, T_{0}(b, c)\right)=f^{-1}\left(f(a) * f\left(f^{-1}(f(b) *\right.\right.$ $f(c)))=f^{-1}(f(a) * f(b) * f(c))=f^{-1}\left(f^{-1}(f(f(a) *\right.$ $f(b)) * f(c)))=T_{0}\left(T_{0}(a, b), c\right)$.

(4) Since $f$ is a strictly monotone decreasing and continuous function, then $f^{-1}$ is also a strictly monotone decreasing and continuous function.

If $a \leq c$ and $b \leq d$, we have $f(a) \geq f(c) \geq 1, f(b) \geq$ $f(d) \geq 1$, and so $f(a) * f(b) \geq f(c) * f(d)$.

Thus, we obtain $f^{-1}(f(a) * f(b)) \leq f^{-1}(f(c) * f(d))$; that is, $T_{0}(a, b) \leq T_{0}(c, d)$.

With the above analysis, the operational laws based on extended $T^{*}$-norm can be derived as below.

Definition 6. Suppose that linguistic terms $s_{\alpha}, s_{\beta} \in \bar{S}=\left\{s_{\alpha} \mid\right.$ $\left.s_{1} \leq s_{\alpha} \leq s_{t}, \alpha \in[1, t]\right\}$, and $\mu \in(0,+\infty)$; the operational laws are defined as below:

$$
\begin{gathered}
s_{\alpha} \otimes s_{\beta}=s_{T_{0}(\alpha, \beta)}=s_{f^{-1}(f(\alpha) * f(\beta))}, \\
\left(s_{\alpha}\right)^{\mu}=s_{f^{-1}\left((f(\alpha))^{\mu}\right)},
\end{gathered}
$$

where $f:[1, t] \rightarrow[1,+\infty)$ is a strictly monotone decreasing and continuous function with $f(1)=+\infty$ and $f(t)=1$.

Theorem 7. Suppose that linguistic terms $s_{\alpha}, s_{\beta}, s_{\chi}, s_{\delta} \in \bar{S}=$ $\left\{s_{\alpha} \mid s_{1} \leq s_{\alpha} \leq s_{t}, \alpha \in[1, t]\right\}$, and $\mu, \mu_{1}, \mu_{2} \in(0,+\infty)$; then the properties of the operational laws are presented as below:

(1) $s_{\alpha} \otimes s_{t}=s_{\alpha}$

(2) $s_{\alpha} \otimes s_{\beta}=s_{\beta} \otimes s_{\alpha}$;

(3) $\left(s_{\alpha} \otimes s_{\beta}\right) \otimes s_{\chi}=s_{\alpha} \otimes\left(s_{\beta} \otimes s_{\chi}\right)$;

(4) $s_{\alpha} \otimes s_{\beta} \geq s_{\chi} \otimes s_{\delta}$, if $\alpha \geq \chi, \beta \geq \delta$;

(5) $\left(s_{\alpha} \otimes s_{\beta}\right)^{\mu}=\left(s_{\alpha}\right)^{\mu} \otimes\left(s_{\beta}\right)^{\mu}$;

(6) $\left(s_{\alpha}\right)^{\mu_{1}} \otimes\left(s_{\alpha}\right)^{\mu_{2}}=\left(s_{\alpha}\right)^{\mu_{1}+\mu_{2}}$;

(7) $\left(s_{\alpha}\right)^{w_{1}} \otimes\left(s_{\alpha}\right)^{w_{2}} \otimes \cdots \otimes\left(s_{\alpha}\right)^{w_{n}}=s_{\alpha}$, if $w_{i} \in[0,1]$ and $\sum_{i=1}^{n} w_{i}=1$.

Proof. (1) Consider $s_{\alpha} \otimes s_{t}=s_{f^{-1}(f(\alpha) * f(t))}=s_{f^{-1}(f(\alpha))}=s_{\alpha}$.

(2) Consider $s_{\alpha} \otimes s_{\beta}=s_{T_{0}(\alpha, \beta)}=s_{f^{-1}(f(\alpha) * f(\beta))}=$ $s_{f^{-1}(f(\beta) * f(\alpha))}=s_{\beta} \otimes s_{\alpha}$.
(3) Consider $\left(s_{\alpha} \otimes s_{\beta}\right) \otimes s_{\chi}=\left(s_{f^{-1}(f(\alpha) * f(\beta))}\right) \otimes$ $s_{\chi}=s_{f^{-1}\left(f\left(f^{-1}(f(\alpha) * f(\beta))\right) * f(\chi)\right)}=s_{f^{-1}(f(\alpha) * f(\beta) * f(\chi))}=$ $s_{f^{-1}\left(f(\alpha) * f\left(f^{-1}(f(\beta) * f(\chi))\right)\right)}=s_{\alpha} \otimes\left(s_{\beta} \otimes s_{\chi}\right)$.

(4) Since $\alpha \geq \chi$ and $\beta \geq \delta$, we have $f(\alpha) * f(\beta) \leq f(\chi) *$ $f(\delta)$, and then $f^{-1}(f(\alpha) * f(\beta)) \geq f^{-1}(f(\chi) * f(\delta))$.

Thus, we obtain $s_{\alpha} \otimes s_{\beta}=s_{f^{-1}(f(\alpha) * f(\beta))} \geq s_{f^{-1}(f(\chi) * f(\delta))}=$ $s_{\chi} \otimes s_{\delta}$.

(5) By (7), we have

$$
\begin{aligned}
\left(s_{\alpha} \otimes s_{\beta}\right)^{\mu} & =\left(s_{f^{-1}(f(\alpha) * f(\beta))}\right)^{\mu} \\
& ={ }_{f^{-1}\left(\left(f\left(f^{-1}(f(\alpha) * f(\beta))\right)\right)^{\mu}\right)}=s_{f^{-1}\left((f(\alpha) * f(\beta))^{\mu}\right)} \\
& =s_{f^{-1}\left((f(\alpha) * f(\beta))^{\mu}\right)}=s_{f^{-1}\left((f(\alpha))^{\mu} *(f(\beta))^{\mu}\right)} \\
& =s_{f^{-1}\left(f\left(f^{-1}\left((f(\alpha))^{\mu}\right)\right) * f\left(f^{-1}\left((f(\beta))^{\mu}\right)\right)\right)} \\
& =s_{f^{-1}\left((f(\alpha))^{\mu}\right)} \otimes s_{f^{-1}\left((f(\beta))^{\mu}\right)} \\
& =\left(s_{\alpha}\right)^{\mu} \otimes\left(s_{\beta}\right)^{\mu} .
\end{aligned}
$$

(6) By (7), we have

$$
\begin{aligned}
\left(s_{\alpha}\right)^{\mu_{1}} & \otimes\left(s_{\alpha}\right)^{\mu_{2}} \\
& =s_{f^{-1}\left((f(\alpha))^{\mu_{1}}\right)} \otimes s_{f^{-1}\left((f(\alpha))^{\mu_{2}}\right)} \\
& =s_{f^{-1}\left(f\left(f^{-1}\left((f(\alpha))^{\mu_{1}}\right)\right) * f\left(f^{-1}\left((f(\alpha))^{\mu_{2}}\right)\right)\right)} \\
& =s_{f^{-1}\left((f(\alpha))^{\mu_{1}} *(f(\alpha))^{\mu_{2}}\right)} \\
& =s_{f^{-1}\left((f(\alpha))^{\mu_{1}+\mu_{2}}\right)}=\left(s_{\alpha}\right)^{\mu_{1}+\mu_{2}} .
\end{aligned}
$$

(7) By (7), we have

$$
\begin{aligned}
\left(s_{\alpha}\right)^{w_{1}} \otimes\left(s_{\alpha}\right)^{w_{2}} \otimes \cdots \otimes\left(s_{\alpha}\right)^{w_{n}} \\
\quad=s_{f^{-1}\left((f(\alpha))^{w_{1}}\right)} \otimes s_{f^{-1}\left((f(\alpha))^{w_{2}}\right)} \otimes \cdots \otimes s_{f^{-1}\left((f(\alpha))^{w_{n}}\right)} \\
\quad=s_{f^{-1}\left(f\left(f^{-1}\left((f(\alpha))^{w_{1}}\right)\right) * f\left(f^{-1}\left((f(\alpha))^{w_{2}}\right)\right) * \cdots * f\left(f^{-1}\left((f(\alpha))^{w_{n}}\right)\right)\right)} \\
\quad=s_{f^{-1}\left((f(\alpha))^{w_{1}} *(f(\alpha))^{w_{2}} * \cdots *(f(\alpha))^{w_{n}}\right)} \\
\quad=s_{f^{-1}\left((f(\alpha))^{w_{1}+w_{2}+\cdots+w_{n}}\right)}=s_{f^{-1}(f(\alpha))}=s_{\alpha} .
\end{aligned}
$$

Having the first four properties above, the operator $\otimes$ can be regarded as an extended $T^{*}$-norm in $\bar{S}$.

\section{Some Uncertain Linguistic Geometric Operators Based on Extended $T^{*}$-Norm}

The operational laws discussed in Section 2 can be extended to UL environment.

Definition 8. Suppose that $\widetilde{s}_{1}=\left[s_{\alpha_{1}}, s_{\beta_{1}}\right]$ and $\widetilde{s}_{2}=\left[s_{\alpha_{2}}, s_{\beta_{2}}\right]$ are two ULVs and $\mu \in(0,+\infty)$; the operational laws are expressed as below: 


$$
\begin{gathered}
\widetilde{s}_{1} \otimes \widetilde{s}_{2}=\left[s_{\alpha_{1}}, s_{\beta_{1}}\right] \otimes\left[s_{\alpha_{2}}, s_{\beta_{2}}\right] \\
=\left[s_{f^{-1}\left(f\left(\alpha_{1}\right) * f\left(\alpha_{2}\right)\right)}, s_{f^{-1}\left(f\left(\beta_{1}\right) * f\left(\beta_{2}\right)\right)}\right], \\
\widetilde{s}_{1}^{\mu}=\left[s_{\alpha_{1}}, s_{\beta_{1}}\right]^{\mu}=\left[s_{f^{-1}\left(\left(f\left(\alpha_{1}\right)\right)^{\mu}\right)}, s_{f^{-1}\left(\left(f\left(\beta_{1}\right)\right)^{\mu}\right)}\right],
\end{gathered}
$$

where $f:[1, t] \rightarrow[1,+\infty)$ is a strictly monotone decreasing and continuous function with $f(1)=+\infty$ and $f(t)=1$.

Similarly, the operational laws in Definition 8 have the properties as below.

Theorem 9. Suppose that $\widetilde{s}_{1}=\left[s_{\alpha_{1}}, s_{\beta_{1}}\right], \widetilde{s}_{2}=\left[s_{\alpha_{2}}, s_{\beta_{2}}\right]$, $\widetilde{s}_{3}=\left[s_{\alpha_{3}}, s_{\beta_{3}}\right]$, and $\widetilde{s}_{4}=\left[s_{\alpha_{4}}, s_{\beta_{4}}\right]$ are ULVs derived from the continuous LTS $\bar{S}=\left\{s_{\alpha} \mid s_{1} \leq s_{\alpha} \leq s_{t}, \alpha \in[1, t]\right\}$, and $\mu, \mu_{1}, \mu_{2} \in(0,+\infty)$; then some properties of the operational laws are presented as below:

(1) $\left[s_{\alpha_{1}}, s_{\beta_{1}}\right] \otimes\left[s_{t}, s_{t}\right]=\left[s_{\alpha_{1}}, s_{\beta_{1}}\right]$;

(2) $\widetilde{s}_{1} \otimes \widetilde{s}_{2}=\widetilde{s}_{2} \otimes \widetilde{s}_{1}$;

(3) $\left(\widetilde{s}_{1} \otimes \widetilde{s}_{2}\right) \otimes \widetilde{s}_{3}=\widetilde{s}_{1} \otimes\left(\widetilde{s}_{2} \otimes \widetilde{s}_{3}\right)$;

(4) $\widetilde{s}_{1} \otimes \widetilde{s}_{2}>\widetilde{s}_{3} \otimes \widetilde{s}_{4}$, if $\alpha_{1} \geq \alpha_{3}, \beta_{1} \geq \beta_{3}, \alpha_{2} \geq \alpha_{4}$, and $\beta_{2} \geq \beta_{4}$

(5) $\left(\widetilde{s}_{1} \otimes \widetilde{s}_{2}\right)^{\mu}=\widetilde{s}_{1}{ }^{\mu} \otimes \widetilde{s}_{2}{ }^{\mu}$;

(6) $\widetilde{s}_{1}{ }^{\mu_{1}} \otimes \widetilde{s}_{1}{ }^{\mu_{2}}=\widetilde{s}_{1}{ }^{\mu_{1}+\mu_{2}}$;

(7) $\widetilde{s}_{1}^{w_{1}} \otimes \widetilde{s}_{1}^{w_{2}} \otimes \cdots \otimes \widetilde{s}_{1}^{w_{n}}=\widetilde{s}_{1}$, if $w_{i} \in[0,1]$ and $\sum_{i=1}^{n} w_{i}=$ 1.

Proof. We only prove (4), (5), and (6).

(4) By (11), we have

$$
\begin{aligned}
\widetilde{s}_{1} \otimes \widetilde{s}_{2} & =\left[s_{\alpha_{1}}, s_{\beta_{1}}\right] \otimes\left[s_{\alpha_{2}}, s_{\beta_{2}}\right] \\
& =\left[s_{f^{-1}\left(f\left(\alpha_{1}\right) * f\left(\alpha_{2}\right)\right)}, s_{f^{-1}\left(f\left(\beta_{1}\right) * f\left(\beta_{2}\right)\right)}\right], \\
\widetilde{s}_{3} \otimes \widetilde{s}_{4} & =\left[s_{\alpha_{3}}, s_{\beta_{3}}\right] \otimes\left[s_{\alpha_{4}}, s_{\beta_{4}}\right] \\
& =\left[s_{f^{-1}\left(f\left(\alpha_{3}\right) * f\left(\alpha_{4}\right)\right)}, s_{f^{-1}\left(f\left(\beta_{3}\right) * f\left(\beta_{4}\right)\right)}\right] .
\end{aligned}
$$

Since both $f$ and $f^{-1}$ are strictly monotone decreasing functions, we have

$$
\begin{aligned}
& f^{-1}\left(f\left(\alpha_{1}\right) * f\left(\alpha_{2}\right)\right) \geq f^{-1}\left(f\left(\alpha_{3}\right) * f\left(\alpha_{4}\right)\right), \\
& f^{-1}\left(f\left(\beta_{1}\right) * f\left(\beta_{2}\right)\right) \geq f^{-1}\left(f\left(\beta_{3}\right) * f\left(\beta_{4}\right)\right) .
\end{aligned}
$$

Let $a=f^{-1}\left(f\left(\alpha_{1}\right) * f\left(\alpha_{2}\right)\right), b=f^{-1}\left(f\left(\beta_{1}\right) * f\left(\beta_{2}\right)\right)$, $c=f^{-1}\left(f\left(\alpha_{3}\right) * f\left(\alpha_{4}\right)\right)$, and $d=f^{-1}\left(f\left(\beta_{3}\right) * f\left(\beta_{4}\right)\right)$; then we have len $\left(\widetilde{s}_{1} \otimes \widetilde{s}_{2}\right)=b-a$ and $\operatorname{len}\left(\widetilde{s}_{3} \otimes \widetilde{s}_{4}\right)=c-d$.

If $d \leq a$, then we have $\max (d-a, 0)=0$. Thus, by (3), we derive $p\left(\widetilde{s}_{1} \otimes \widetilde{s}_{2} \geq \widetilde{s}_{3} \otimes \widetilde{s}_{4}\right)=1$.

If $d \geq a$, we can easily get $\max (d-a, 0)=d-a \leq$ $(1 / 2)\left(\operatorname{len}\left(\widetilde{s}_{1} \otimes \widetilde{s}_{2}\right)+\operatorname{len}\left(\widetilde{s}_{3} \otimes \widetilde{s}_{4}\right)\right)$, so we have $p\left(\widetilde{s}_{1} \otimes \widetilde{s}_{2}>\widetilde{s}_{3} \otimes \widetilde{s}_{4}\right) \geq$ 0.5 .

Consequently, we obtain $\widetilde{s}_{1} \otimes \widetilde{s}_{2}>\widetilde{s}_{3} \otimes \widetilde{s}_{4}$.
(5) By (11) and (12), we have

$$
\begin{aligned}
& \left(\widetilde{s}_{1} \otimes \widetilde{s}_{2}\right)^{\mu}=\left[s_{f^{-1}\left(f\left(\alpha_{1}\right) * f\left(\alpha_{2}\right)\right)}, s_{f^{-1}\left(f\left(\beta_{1}\right) * f\left(\beta_{2}\right)\right)}\right]^{\mu} \\
& =\left[s_{f^{-1}\left(\left(f\left(f^{-1}\left(f\left(\alpha_{1}\right) * f\left(\alpha_{2}\right)\right)\right)\right)^{u}\right)},\right. \\
& \left.s_{f^{-1}\left(\left(f\left(f^{-1}\left(f\left(\beta_{1}\right) * f\left(\beta_{2}\right)\right)\right)\right)^{u}\right)}\right] \\
& =\left[s_{f^{-1}\left(\left(f\left(\alpha_{1}\right) * f\left(\alpha_{2}\right)\right)^{u}\right)}, s_{f^{-1}\left(\left(f\left(\beta_{1}\right) * f\left(\beta_{2}\right)\right)^{u}\right)}\right] \\
& =\left[s_{f^{-1}\left(\left(f\left(\alpha_{1}\right)\right)^{\mu} *\left(f\left(\alpha_{2}\right)\right)^{\mu}\right)}, s_{f^{-1}\left(\left(f\left(\beta_{1}\right)\right)^{\mu} *\left(f\left(\beta_{2}\right)\right)^{\mu}\right)}\right] \\
& =\left[s_{f^{-1}\left(f\left(f^{-1}\left(\left(f\left(\alpha_{1}\right)\right)^{\mu}\right)\right) * f\left(f^{-1}\left(\left(f\left(\alpha_{2}\right)\right)^{\mu}\right)\right)\right)},\right.
\end{aligned}
$$

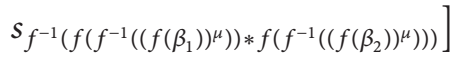

$$
\begin{aligned}
& =\left[s_{f^{-1}\left(\left(f\left(\alpha_{1}\right)\right)^{\mu}\right)}, s_{f^{-1}\left(\left(f\left(\beta_{1}\right)\right)^{\mu}\right)}\right] \\
& \otimes\left[s_{f^{-1}\left(\left(f\left(\alpha_{2}\right)\right)^{\mu}\right)}, s_{f^{-1}\left(\left(f\left(\beta_{2}\right)\right)^{\mu}\right)}\right]=\widetilde{s}_{1}{ }^{\mu} \otimes \widetilde{s}_{2}{ }^{\mu} .
\end{aligned}
$$

(6) By (11) and (12), we have

$$
\begin{aligned}
\widetilde{s}_{1}^{\mu_{1}} \otimes \widetilde{s}_{1}^{\mu_{2}}= & {\left[s_{f^{-1}\left(\left(f\left(\alpha_{1}\right)\right)^{\mu_{1}}\right)}, s_{\left.f^{-1}\left(\left(f\left(\beta_{1}\right)\right)^{\mu_{1}}\right)\right]}\left[s_{f^{-1}\left(\left(f\left(\alpha_{1}\right)\right)^{\mu_{2}}\right)}, s_{f^{-1}\left(\left(f\left(\beta_{1}\right)\right)^{\mu_{2}}\right)}\right]\right.} \\
= & {\left[s_{f^{-1}\left(f\left(f^{-1}\left(\left(f\left(\alpha_{1}\right)\right)^{\mu_{1}}\right)\right) * f\left(f^{-1}\left(\left(f\left(\alpha_{1}\right)\right)^{\mu_{2}}\right)\right)\right),}\right.} \\
& \left.s_{f^{-1}\left(f\left(f^{-1}\left(\left(f\left(\beta_{1}\right)\right)^{\mu_{1}}\right)\right) * f\left(f^{-1}\left(\left(f\left(\beta_{1}\right)\right)^{\mu_{2}}\right)\right)\right)}\right] \\
= & {\left[s_{f^{-1}\left(\left(f\left(\alpha_{1}\right)\right)^{\mu_{1}} *\left(f\left(\alpha_{1}\right)\right)^{\mu_{2}}\right),}\right.} \\
= & {\left[s_{f^{-1}\left(\left(f\left(\beta_{1}\right)\right)^{\mu_{1}} *\left(f\left(\beta_{1}\right)\right)^{\mu_{2}}\right)}\right] } \\
= & \widetilde{s}_{1}^{\mu_{1}+\mu_{2}} .
\end{aligned}
$$

Example 10. Let $\bar{S}=\left\{s_{\alpha} \mid s_{1} \leq s_{\alpha} \leq s_{9}, \alpha \in[1,9]\right\}, \widetilde{s}_{1}=$ $\left[s_{3}, s_{4}\right], \widetilde{s}_{2}=\left[s_{4}, s_{6}\right], \widetilde{s}_{3}=\left[s_{5}, s_{8}\right], \mu=3$, and $f(x)=(t-$ $1) /(x-1)=8 /(x-1)$; then $f^{-1}(x)=(x+t-1) / x=(x+8) / x$. By (11) and (12), we have

$$
\begin{aligned}
\widetilde{s}_{1} \otimes \widetilde{s}_{2} & =\left[s_{3}, s_{4}\right] \otimes\left[s_{4}, s_{6}\right] \\
& =\left[s_{f^{-1}(f(3) * f(4))}, s_{f^{-1}(f(4) * f(6))}\right] \\
& =\left[s_{1.75}, s_{2.875}\right] \\
\widetilde{s}_{1} \otimes \widetilde{s}_{3} & =\left[s_{3}, s_{4}\right] \otimes\left[s_{5}, s_{8}\right] \\
& =\left[s_{f^{-1}(f(3) * f(5))}, s_{f^{-1}(f(4) * f(8))}\right] \\
& =\left[s_{2}, s_{3.625}\right],
\end{aligned}
$$




$$
\begin{aligned}
\widetilde{s}_{1}^{\mu} & =\left[s_{3}, s_{4}\right]^{3}=\left[s_{f^{-1}\left((f(3))^{3}\right)}, s_{f^{-1}\left((f(4))^{3}\right)}\right] \\
& =\left[s_{1.125}, s_{1.422}\right] .
\end{aligned}
$$

It is easy to see that $\widetilde{s}_{1} \prec \widetilde{s}_{2} \prec \widetilde{s}_{3}$ and $\widetilde{s}_{1} \otimes \widetilde{s}_{2} \prec \widetilde{s}_{1} \otimes \widetilde{s}_{3}$.

However, if we follow the operational laws defined by (2), then we have

$$
\begin{aligned}
& \widetilde{s}_{1} \otimes \widetilde{s}_{2}=\left[s_{3}, s_{4}\right] \otimes\left[s_{4}, s_{6}\right]=\left[s_{12}, s_{24}\right], \\
& \widetilde{s}_{1} \otimes \widetilde{s}_{3}=\left[s_{3}, s_{4}\right] \otimes\left[s_{5}, s_{8}\right]=\left[s_{15}, s_{32}\right] .
\end{aligned}
$$

Obviously, the computing results are beyond the discourse domain of the linguistic variable. Alternatively, if we follow the method of $\mathrm{Xu}$ [14], then we have

$$
\begin{aligned}
\widetilde{s}_{1} \otimes \widetilde{s}_{2} & =\left[s_{3}, s_{4}\right] \otimes\left[s_{4}, s_{6}\right] \\
& =\left[\min \left\{s_{3 \times 4}, s_{9}\right\}, \min \left\{s_{4 \times 6}, s_{9}\right\}\right] \\
& =\left[s_{9}, s_{9}\right], \\
\widetilde{s}_{1} \otimes \widetilde{s}_{3} & =\left[s_{3}, s_{4}\right] \otimes\left[s_{5}, s_{8}\right] \\
& =\left[\min \left\{s_{3 \times 5}, s_{9}\right\}, \min \left\{s_{4 \times 8}, s_{9}\right\}\right] \\
& =\left[s_{9}, s_{9}\right] .
\end{aligned}
$$

That is, $\widetilde{s}_{1} \otimes \widetilde{s}_{2}=\widetilde{s}_{1} \otimes \widetilde{s}_{3}$, which seem to be counterintuitive and may not be easily accepted.

On the basis of the operational laws of ULVs, in what follows, we propose some extended UL geometric operators.

Definition 11. Suppose that $\widetilde{s}_{j}=\left[s_{\alpha_{j}}, s_{\beta_{j}}\right](j=1,2, \ldots, m)$ are a series of ULVs; then the extended UL geometric mean (EULGM) operator is defined as

$$
\operatorname{EULGM}\left(\widetilde{s}_{1}, \widetilde{s}_{2}, \ldots, \widetilde{s}_{m}\right)=\left(\widetilde{s}_{1} \otimes \widetilde{s}_{2} \otimes \cdots \otimes \widetilde{s}_{m}\right)^{1 / m} .
$$

The EULGM operator has the following properties.

Theorem 12. Suppose that $\widetilde{s}_{j}=\left[s_{\alpha_{j}}, s_{\beta_{j}}\right](j=1,2, \ldots, m)$ are a series of ULVs; then one has

(1) idempotency: if $\widetilde{s}_{j}=\left[s_{\alpha_{j}}, s_{\beta_{j}}\right]=\widetilde{s}=\left[s_{\alpha}, s_{\beta}\right], j=$ $1,2, \ldots, m$, then

$$
\operatorname{EULGM}\left(\widetilde{s}_{1}, \widetilde{s}_{2}, \ldots, \widetilde{s}_{m}\right)=\widetilde{s} ;
$$

(2) monotonicity: suppose that $\widetilde{s}_{j}^{\prime}=\left[s^{\prime}{ }_{\alpha_{j}}, s_{\beta_{j}}^{\prime}\right](j=1,2$, $\ldots, m)$ are a series of ULVs. If $s_{\alpha_{j}}^{\prime} \geq s_{\alpha_{j}}$ and $s_{\beta_{j}}^{\prime} \geq$ $s_{\beta_{j}}(j=1,2, \ldots, m)$, then

$\operatorname{EULGM}\left(\widetilde{s}_{1}^{\prime}, \widetilde{s}_{2}^{\prime}, \ldots, \widetilde{s}_{m}^{\prime}\right)>\operatorname{EULGM}\left(\widetilde{s}_{1}, \widetilde{s}_{2}, \ldots, \widetilde{s}_{m}\right) ;$

(3) boundary:

$$
\begin{aligned}
{\left[\min _{j} s_{\alpha_{j}}, \min _{j} s_{\beta_{j}}\right] } & \prec \operatorname{EULGM}\left(\widetilde{s}_{1}, \widetilde{s}_{2}, \ldots, \widetilde{s}_{m}\right) \\
& \prec\left[\max _{j} s_{\alpha_{j}}, \max _{j} s_{\beta_{j}}\right] ;
\end{aligned}
$$

(4) commutativity: suppose that $\left(\widetilde{s}_{1}^{\prime}, \widetilde{s}_{2}^{\prime}, \ldots, \widetilde{s}_{m}^{\prime}\right)$ is any permutation of $\left(\widetilde{s}_{1}, \widetilde{s}_{2}, \ldots, \widetilde{s}_{m}\right)$; then

$$
\operatorname{EULGM}\left(\widetilde{s}_{1}^{\prime}, \widetilde{s}_{2}^{\prime}, \ldots, \widetilde{s}_{m}^{\prime}\right)=\operatorname{EULGM}\left(\widetilde{s}_{1}, \widetilde{s}_{2}, \ldots, \widetilde{s}_{m}\right) .
$$

Definition 13. Suppose that $\widetilde{s}_{j}=\left[s_{\alpha_{j}}, s_{\beta_{j}}\right](j=1,2, \ldots, m)$ are a series of ULVs, and $w=\left(w_{1}, w_{2}, \ldots, w_{m}\right)^{T}$ (satisfying $0 \leq$ $w_{j} \leq 1$ and $\left.\sum_{j=1}^{m} w_{j}=1\right)$ is the weight vector regarding ULVs; then the extended UL weighted geometric mean (EULWGM) operator is defined as

$$
\operatorname{EULWGM}\left(\widetilde{s}_{1}, \widetilde{s}_{2}, \ldots, \widetilde{s}_{m}\right)=\widetilde{s}_{1}^{w_{1}} \otimes \widetilde{s}_{2}^{w_{2}} \otimes \cdots \otimes \widetilde{s}_{m}^{w_{m}} .
$$

If $w_{j}=1 / n, j=1,2, \ldots, m$, then the EULGWM operator degenerates to the EULGM operator. The EULWGM operator has the properties as below.

Theorem 14. Suppose that $\widetilde{s}_{j}=\left[s_{\alpha_{j}}, s_{\beta_{j}}\right](j=1,2, \ldots, m)$ are a series of ULVs, and $w=\left(w_{1}, w_{2}, \ldots, w_{m}\right)^{T}$ (satisfying $0 \leq$ $w_{j} \leq 1$ and $\left.\sum_{j=1}^{m} w_{j}=1\right)$ is the weight vector regarding ULVs; then one derives

(1) idempotency: if $\widetilde{s}_{j}=\left[s_{\alpha_{j}}, s_{\beta_{j}}\right]=\widetilde{s}=\left[s_{\alpha}, s_{\beta}\right], j=$ $1,2, \ldots, m$, then

$$
\operatorname{EULWGM}\left(\widetilde{s}_{1}, \widetilde{s}_{2}, \ldots, \widetilde{s}_{m}\right)=\widetilde{s} ;
$$

(2) monotonicity: suppose that $\tilde{s}_{j}^{\prime}=\left[s^{\prime}{ }_{\alpha_{j}}, s_{\beta_{j}}^{\prime}\right](j=1,2$, $\ldots, m)$ are a series of ULVs. If $s_{\alpha_{j}}^{\prime} \geq s_{\alpha_{j}}$ and $s_{\beta_{j}}^{\prime} \geq$ $s_{\beta_{j}}(j=1,2, \ldots, m)$, then

$$
\operatorname{EULWGM}\left(\widetilde{s}_{1}^{\prime},{ }_{2} \widetilde{s}_{2}^{\prime}, \ldots, \widetilde{s}_{m}^{\prime}\right)>\operatorname{EULWGM}\left(\widetilde{s}_{1}, \widetilde{s}_{2}, \ldots, \widetilde{s}_{m}\right) ;
$$

(3) boundary:

$$
\begin{aligned}
{\left[\min _{j} s_{\alpha_{j}}, \min _{j} s_{\beta_{j}}\right] } & \prec \operatorname{EULWGM}\left(\widetilde{s}_{1}, \widetilde{s}_{2}, \ldots, \widetilde{s}_{m}\right) \\
& \prec\left[\max _{j} s_{\alpha_{j}}, \max _{j} s_{\beta_{j}}\right] .
\end{aligned}
$$

Definition 15. Suppose that $\widetilde{s}_{j}=\left[s_{\alpha_{j}}, s_{\beta_{j}}\right](j=1,2, \ldots, m)$ are a series of ULVs; then the extended UL ordered weighted geometric (EULOGW) operator is defined as

$$
\operatorname{EULOWG}\left(\widetilde{s}_{1}, \widetilde{s}_{2}, \ldots, \widetilde{s}_{m}\right)=\widetilde{s}_{(1)}^{w_{1}} \otimes \widetilde{s}_{(2)}^{w_{2}} \otimes \cdots \otimes \widetilde{s}_{(m)}^{w_{m}},
$$

where $\widetilde{s}_{(j)}$ is the $j$ th largest $\widetilde{s}_{i}$ and $w=\left(w_{1}, w_{2}, \ldots, w_{m}\right)^{T}$ (satisfying $0 \leq w_{j} \leq 1$ and $\sum_{j=1}^{m} w_{j}=1$ ) is the aggregationassociated vector.

Theorem 16. Suppose that $\widetilde{s}_{j}=\left[s_{\alpha_{j}}, s_{\beta_{j}}\right](j=1,2, \ldots, m)$ are a series of ULVs; then one derives

(1) idempotency: if $\widetilde{s}_{j}=\left[s_{\alpha_{j}}, s_{\beta_{j}}\right]=\widetilde{s}=\left[s_{\alpha}, s_{\beta}\right], j=$ $1,2, \ldots, m$, then

$$
\operatorname{EULOWG}\left(\widetilde{s}_{1}, \widetilde{s}_{2}, \ldots, \widetilde{s}_{m}\right)=\widetilde{s} ;
$$


(2) monotonicity: suppose that $\widetilde{s}_{j}^{\prime}=\left[s_{\alpha_{j}}^{\prime}, s_{\beta_{j}}^{\prime}\right](j=1,2, \ldots$, $m)$ are a series of ULVs. If $s_{\alpha_{j}}^{\prime} \geq s_{\alpha_{j}}$ and $s_{\beta_{j}}^{\prime} \geq s_{\beta_{j}}(j=$ $1,2, \ldots, m)$, then

$$
\operatorname{EULOWG}\left(\widetilde{s}_{1}^{\prime}, \widetilde{s}_{2}^{\prime}, \ldots, \widetilde{s}_{m}^{\prime}\right)>\operatorname{EULOWG}\left(\widetilde{s}_{1}, \widetilde{s}_{2}, \ldots, \widetilde{s}_{m}\right)
$$

(3) boundary:

$$
\begin{aligned}
{\left[\operatorname{mins}_{j}, \min _{j} s_{\beta_{j}}\right] } & \prec \operatorname{EULOWG}\left(\widetilde{s}_{1}, \widetilde{s}_{2}, \ldots, \widetilde{s}_{m}\right) \\
& \prec\left[\max _{j} s_{\alpha_{j}}, \max _{j} s_{\beta_{j}}\right] ;
\end{aligned}
$$

(4) commutativity: suppose that $\left(\widetilde{s}_{1}^{\prime}, \widetilde{s}_{2}^{\prime}, \ldots, \widetilde{s}_{m}^{\prime}\right)$ is any permutation of $\left(\widetilde{s}_{1}, \widetilde{s}_{2}, \ldots, \widetilde{s}_{m}\right)$; then

$$
\operatorname{EULOWG}\left(\widetilde{s}_{1}^{\prime}, \widetilde{s}_{2}^{\prime}, \ldots, \widetilde{s}_{m}^{\prime}\right)=\operatorname{EULOWG}\left(\widetilde{s}_{1}, \widetilde{s}_{2}, \ldots, \widetilde{s}_{m}\right) \text {. }
$$

The above UL aggregation operators do not take the relationship between the ULVs being aggregated into account. To avoid such limitation, Yager [21] proposed the PA operator which allows argument values to support each other in the aggregation process. In addition, $\mathrm{Xu}$ and Yager [22] developed the PG operator, weighted PG operator, and POWG operator. On the basis of the operational laws of ULVs, in what follows, we introduce some PG operators for ULVs.

Definition 17. Suppose that $\widetilde{s}_{j}=\left[s_{\alpha_{j}}, s_{\beta_{j}}\right](j=1,2, \ldots, m)$ are a series of ULVs; then the extended UL PG (EULPG) operator is defined as

$$
\operatorname{EULPG}\left(\widetilde{s}_{1}, \widetilde{s}_{2}, \ldots, \widetilde{s}_{m}\right)=\prod_{i=1}^{m} \widetilde{s}_{i}^{\left(1+T\left(\tilde{s}_{i}\right)\right) / \sum_{i=1}^{n}\left(1+T\left(\widetilde{s}_{i}\right)\right)},
$$

where $T\left(\widetilde{s}_{i}\right)=\sum_{j=1, j \neq i}^{m} \operatorname{Su}\left(\widetilde{s}_{i}, \widetilde{s}_{j}\right)$ and $\operatorname{Su}\left(\widetilde{s}_{i}, \widetilde{s}_{j}\right)$ is the support for $\widetilde{s}_{i}$ from $\widetilde{s}_{j}$, which meets the following properties [22]:

(1) $0 \leq \operatorname{Su}\left(\widetilde{s}_{i}, \widetilde{s}_{j}\right) \leq 1$;

(2) $\operatorname{Su}\left(\widetilde{s}_{i}, \widetilde{s}_{j}\right)=\operatorname{Su}\left(\widetilde{s}_{j}, \widetilde{s}_{i}\right)$;

(3) $\operatorname{Su}\left(\widetilde{s}_{i}, \widetilde{s}_{j}\right) \geq \operatorname{Su}\left(\widetilde{s}_{k}, \widetilde{s}_{l}\right)$, if $S\left(\widetilde{s}_{i}, \widetilde{s}_{j}\right) \geq S\left(\widetilde{s}_{k}, \widetilde{s}_{l}\right)$, where $S$ is a similarity measure between ULVs.

In this paper, we defined $\operatorname{Su}\left(\widetilde{s}_{i}, \widetilde{s}_{j}\right)$ as $S\left(\widetilde{s}_{i}, \widetilde{s}_{j}\right)$. Without loss of generality, the similarity measure between $\widetilde{s}_{i}=\left[s_{\alpha_{i}}, s_{\beta_{i}}\right]$ and $\widetilde{s}_{j}=\left[s_{\alpha_{j}}, s_{\beta_{j}}\right]$ is determined based on Hamming distance; that is,

$$
\operatorname{Su}\left(\widetilde{s}_{i}, \widetilde{s}_{j}\right)=S\left(\widetilde{s}_{i}, \widetilde{s}_{j}\right)=1-\frac{\left|\alpha_{i}-\alpha_{j}\right|+\left|\beta_{i}-\beta_{j}\right|}{2(t-1)},
$$

where $\widetilde{s}_{i}$ and $\widetilde{s}_{j}$ are derived from the continuous LTS $\bar{S}=\left\{s_{\alpha} \mid\right.$ $\left.s_{1} \leq s_{\alpha} \leq s_{t}, \alpha \in[1, t]\right\}$.

Obviously, $S\left(\widetilde{s}_{i}, \widetilde{s}_{j}\right) \in[0,1]$, where $S\left(\widetilde{s}_{i}, \widetilde{s}_{j}\right)=1$ if and only if $\alpha_{i}=\alpha_{j}$ and $\beta_{i}=\beta_{j}$, and $S\left(\widetilde{s}_{i}, \widetilde{s}_{j}\right)=0$ if and only if $\left|\alpha_{i}-\alpha_{j}\right|=\left|\beta_{i}-\beta_{j}\right|=t-1$; that is, $\widetilde{s}_{i}=\left[s_{1}, s_{1}\right]$ and $\widetilde{s}_{j}=\left[s_{t}, s_{t}\right]$ (or $\widetilde{s}_{i}=\left[s_{t}, s_{t}\right]$ and $\widetilde{s}_{j}=\left[s_{1}, s_{1}\right]$ ), which means there is no support between $\widetilde{s}_{i}$ and $\widetilde{s}_{j}$ in the geometric aggregation process. In addition, if all the $\operatorname{Su}\left(\widetilde{s}_{i}, \widetilde{s}_{j}\right)$ are equal, then the EULPG operator degenerates to the EULGM operator.
Theorem 18. Suppose that $\widetilde{s}_{j}=\left[s_{\alpha_{j}}, s_{\beta_{j}}\right](j=1,2, \ldots, m)$ are a series of ULVs; then one derives

(1) idempotency: if $\widetilde{s}_{j}=\left[s_{\alpha_{j}}, s_{\beta_{j}}\right]=\widetilde{s}=\left[s_{\alpha}, s_{\beta}\right], j=$ $1,2, \ldots, m$, then

$$
\operatorname{EULPG}\left(\widetilde{s}_{1}, \widetilde{s}_{2}, \ldots, \widetilde{s}_{m}\right)=\widetilde{s}
$$

(2) boundary:

$$
\begin{aligned}
{\left[\min _{j} s_{\alpha_{j}}, \min _{j} s_{\beta_{j}}\right] } & \prec \operatorname{EULPG}\left(\widetilde{s}_{1}, \widetilde{s}_{2}, \ldots, \widetilde{s}_{m}\right) \\
& \prec\left[\max _{j} s_{\alpha_{j}}, \max _{j} s_{\beta_{j}}\right] ;
\end{aligned}
$$

(3) commutativity: suppose that $\left(\widetilde{s}_{1}^{\prime}, \widetilde{s}_{2}^{\prime}, \ldots, \widetilde{s}_{m}^{\prime}\right)$ is any permutation of $\left(\widetilde{s}_{1}, \widetilde{s}_{2}, \ldots, \widetilde{s}_{m}\right)$; then

$$
\operatorname{EULPG}\left(\widetilde{s}_{1}^{\prime}, \widetilde{s}_{2}^{\prime}, \ldots, \widetilde{s}_{m}^{\prime}\right)=\operatorname{EULPG}\left(\widetilde{s}_{1}, \widetilde{s}_{2}, \ldots, \widetilde{s}_{m}\right)
$$

If the weights of $\widetilde{s}_{j}(j=1,2, \ldots, m)$ in the EULPG operator are taken into account, the extended UL weighted $P G$ (EULWPG) operator is derived.

Definition 19. Suppose that $\widetilde{s}_{j}=\left[s_{\alpha_{j}}, s_{\beta_{j}}\right](j=1,2, \ldots, m)$ are a series of ULVs; then the extended UL weighted PG (EULWPG) operator is defined as

$$
\operatorname{EULWPG}\left(\widetilde{s}_{1}, \widetilde{s}_{2}, \ldots, \widetilde{s}_{m}\right)=\prod_{i=1}^{m} \widetilde{s}_{i} w_{i}\left(1+T\left(\widetilde{s}_{i}\right)\right) / \sum_{i=1}^{n} w_{i}\left(1+T\left(\widetilde{s}_{i}\right)\right),
$$

where $T\left(\widetilde{s}_{i}\right)=\sum_{j=1, j \neq i}^{m} w_{i} \operatorname{Su}\left(\widetilde{s}_{i}, \widetilde{s}_{j}\right)$ and $w_{i}$ is the weight of $\widetilde{s}_{i}$ (satisfying $0 \leq w_{j} \leq 1$ and $\sum_{i=1}^{m} w_{i}=1$ ).

Obviously, the EULWPG operator is not commutative but idempotent and bounded.

Motivated by POWG operator [22], we propose an extended UL power ordered weighted geometric (EULPOWG) operator as below.

Definition 20. Suppose that $\widetilde{s}_{j}=\left[s_{\alpha_{j}}, s_{\beta_{j}}\right](j=1,2, \ldots, m)$ are a series of ULVs; then the extended UL power ordered weighted geometric (EULPOWG) operator is defined as

$$
\operatorname{EULPOWG}\left(\widetilde{s}_{1}, \widetilde{s}_{2}, \ldots, \widetilde{s}_{m}\right)=\prod_{i=1}^{m} \widetilde{s}_{(i)}^{u_{i}},
$$

where $\widetilde{s}_{(i)}$ is the $i$ th largest of $\widetilde{s}_{j}, u_{i}=g\left(R_{i} / T V\right)-g\left(R_{i-1} / T V\right)$, $R_{i}=\sum_{j=1}^{i} V_{j}, T V=\sum_{j=1}^{m} V_{j}, V_{j}=1+T\left(\widetilde{s}_{(j)}\right), T\left(\widetilde{s}_{(j)}\right)=$ $\sum_{i=1, i \neq j}^{m} \operatorname{Su}\left(\widetilde{\boldsymbol{s}}_{(j)}, \widetilde{\boldsymbol{s}}_{(i)}\right), \operatorname{Su}\left(\widetilde{\boldsymbol{s}}_{(j)}, \widetilde{\boldsymbol{s}}_{(i)}\right)$ is the support for $\widetilde{\boldsymbol{s}}_{(j)}$ from $\widetilde{\boldsymbol{s}}_{(i)}$, and $g:[0,1] \rightarrow[0,1]$ is a basic unit-interval monotonic function [22] with the following properties: (1) $g(0)=0$; (2) $g(1)=1 ;(3) g(x) \geq g(y)$, if $x>y$. 
In particular, if $g(x)=x$, then the EULPOWG operator degenerates to the EULPG operator. By (40), we derive

$$
\begin{aligned}
\operatorname{EULPOWG}\left(\widetilde{s}_{1}, \widetilde{s}_{2}, \ldots, \widetilde{s}_{m}\right) & =\prod_{i=1}^{m} \widetilde{r}_{i}^{g\left(R_{i} / T V\right)-g\left(R_{i-1} / T V\right)} \\
& =\prod_{i=1}^{m} \widetilde{r}_{i}^{R_{i} / T V-R_{i-1} / T V}=\prod_{i=1}^{m} \widetilde{r}_{i} V_{i} / T V \\
& =\prod_{i=1}^{m} \widetilde{r}_{i}^{\left(1+T\left(\widetilde{r}_{i}\right)\right) / \sum_{i=1}^{n}\left(1+T\left(\widetilde{r}_{i}\right)\right)} \\
& =\operatorname{EULPG}\left(\widetilde{s}_{1}, \widetilde{s}_{2}, \ldots, \widetilde{s}_{m}\right) .
\end{aligned}
$$

The EULPOWG operator has the properties as below.

Theorem 21. Suppose that $\widetilde{s}_{j}=\left[s_{\alpha_{j}}, s_{\beta_{j}}\right](j=1,2, \ldots, m)$ are a series of ULVs; then one derives

(1) idempotency: if $\widetilde{s}_{j}=\left[s_{\alpha_{j}}, s_{\beta_{j}}\right]=\widetilde{s}=\left[s_{\alpha}, s_{\beta}\right], j=$ $1,2, \ldots, m$, then

$$
\operatorname{EULPOWG}\left(\widetilde{s}_{1}, \widetilde{s}_{2}, \ldots, \widetilde{s}_{m}\right)=\widetilde{s}
$$

(2) boundary:

$$
\begin{aligned}
{\left[\min _{j} s_{\alpha_{j}}, \min _{j} s_{\beta_{j}}\right] } & \prec \operatorname{EULPOWG}\left(\widetilde{s}_{1}, \widetilde{s}_{2}, \ldots, \widetilde{s}_{m}\right) \\
& \prec\left[\max _{j} s_{\alpha_{j}}, \max _{j} s_{\beta_{j}}\right]
\end{aligned}
$$

(3) commutativity: suppose that $\left(\widetilde{s}_{1}^{\prime}, \widetilde{s}_{2}^{\prime}, \ldots, \widetilde{s}_{m}^{\prime}\right)$ is any permutation of $\left(\widetilde{s}_{1}, \widetilde{s}_{2}, \ldots, \widetilde{s}_{m}\right)$; then

$\operatorname{EULPOWG}\left(\widetilde{s}_{1}^{\prime}, \widetilde{s}_{2}^{\prime}, \ldots, \widetilde{s}_{m}^{\prime}\right)=\operatorname{EULPOWG}\left(\widetilde{s}_{1}, \widetilde{s}_{2}, \ldots, \widetilde{s}_{m}\right)$.

\section{MAGDM Method Based on the Extended Uncertain Linguistic Aggregation Operators}

In what follows, a MAGDM method presents the application of the aggregation operators proposed in Section 3.

Let $A=\left(A_{1}, A_{2}, \ldots, A_{m}\right), C=\left(C_{1}, C_{2}, \ldots, C_{n}\right)$, and $D=\left(D_{1}, D_{2}, \ldots, D_{l}\right)$ be the sets of alternatives, attributes, and decision makers, respectively. The attribute weights are known and satisfy $w_{i} \in[0,1]$ and $\sum_{i=1}^{n} w_{i}=1$. Let $R_{k}=$ $\left(r_{i j}^{k}\right)_{m \times n}$ be the decision matrix given by $D_{k}$, where $r_{i j}^{k}=$ $\left[s_{\alpha_{i j}}^{k}, s_{\beta_{i j}}^{k}\right]$ expresses the performance value for $A_{i}$ with respect to $C_{j}$ and takes the form of ULV with $s_{\alpha_{i j}}^{k}, s_{\beta_{i j}}^{k} \in S=\left\{s_{i} \mid i=\right.$ $1, \ldots, t\}$.
The main processes of the method proposed are described as below.

Step 1. Each decision maker is asked to provide the UL decision matrix $R_{k}=\left(r_{i j}^{k}\right)_{m \times n}$.

Step 2. By the EULPOWG operator, the aggregated decision matrix $R=\left(r_{i j}\right)_{m \times n}$ can be derived; that is, $r_{i j}=\left[s_{\alpha_{i j}}, s_{\beta_{i j}}\right]=$ $\operatorname{EULPOWG}\left(r_{i j}^{1}, r_{i j}^{2}, \ldots, r_{i j}^{l}\right)$.

Step 3. Aggregate $r_{i j}$ to obtain the overall performance values $r_{i}$ for each alternative $A_{i}(i=1,2, \ldots, m)$ based on the EULWGM operator.

Step 4. Obtain the order of the alternatives by Definition 1.

\section{Application Example}

To demonstrate the application of the proposed model, in this section, we provide an example where the decision makers from a manufacturing company have to select a third-party logistics provider.

With the rapid change of competitive environment, more and more companies focus on their core competency and logistics outsourcing has become an important strategy to promote enterprise's competitiveness. Within new strategies for purchasing and manufacturing, third-party logistics providers play a key role in achieving corporate competition. Particularly for manufacturing companies, the selection of appropriate third-party logistics provider is of high importance. Sustainable third-party logistics provider selection requires the evaluation of providers' performance from several metrics. That is, in the first stage of third-party logistics provider selection, the manufacturing company should define the qualitative and quantitative attributes which are taken into account to evaluate and select a supplier. After full consideration of the long-term strategy, the manufacturing company constructs the following evaluation systems including four aspects (suppose the weight vector is $w=$ $(0.3,0.1,0.2,0.4)): C_{1}$ is the innovation capability; $C_{2}$ is the sense of social responsibility and environment performance; $C_{3}$ is the ability of sustainability; $C_{4}$ is the quality of service. Then, the manufacturing company organizes a committee of experts who are responsible for providing their evaluations on each supplier with respect to each attribute. Suppose there are four third-party logistics providers (alternatives: $A_{1}, A_{2}$, $A_{3}$, and $A_{4}$ ) to be evaluated. In addition, three experts take part in the decision making and give their evaluations with the LTS $S=\left\{s_{1}=\right.$ extremely poor, $s_{2}=$ very poor, $s_{3}=$ poor, $s_{4}=$ slightly poor, $s_{5}=$ fair, $s_{6}=$ slightly good, $s_{7}=$ good, $s_{8}=$ very good, $s_{9}=$ extremely good $\}$.

In the existing research on third-party logistics provider or supplier selection models, although the criteria used by most of them and performance values given by decision makers are not completely independent, the dependent and feedback effects are often neglected. In what follows, we applied the extended UL geometric aggregation operators to solve the above MAGDM problem. 
TABLE 1: The decision matrix $R_{1}$ given by $D_{1}$.

\begin{tabular}{lcccc}
\hline & $C_{1}$ & $C_{2}$ & $C_{3}$ & $C_{4}$ \\
\hline$A_{1}$ & {$\left[s_{4}, s_{7}\right]$} & {$\left[s_{6}, s_{7}\right]$} & {$\left[s_{1}, s_{3}\right]$} & {$\left[s_{3}, s_{5}\right]$} \\
$A_{2}$ & {$\left[s_{5}, s_{7}\right]$} & {$\left[s_{2}, s_{4}\right]$} & {$\left[s_{3}, s_{4}\right]$} & {$\left[s_{4}, s_{6}\right]$} \\
$A_{3}$ & {$\left[s_{7}, s_{8}\right]$} & {$\left[s_{3}, s_{4}\right]$} & {$\left[s_{3}, s_{6}\right]$} & {$\left[s_{4}, s_{5}\right]$} \\
$A_{4}$ & {$\left[s_{3}, s_{3}\right]$} & {$\left[s_{1}, s_{2}\right]$} & {$\left[s_{4}, s_{4}\right]$} & {$\left[s_{3}, s_{4}\right]$} \\
\hline
\end{tabular}

TABLE 2: The decision matrix $R_{2}$ given by $D_{2}$.

\begin{tabular}{lcccc}
\hline & $C_{1}$ & $C_{2}$ & $C_{3}$ & $C_{4}$ \\
\hline$A_{1}$ & {$\left[s_{3}, s_{4}\right]$} & {$\left[s_{1}, s_{2}\right]$} & {$\left[s_{3}, s_{4}\right]$} & {$\left[s_{3}, s_{4}\right]$} \\
$A_{2}$ & {$\left[s_{3}, s_{5}\right]$} & {$\left[s_{4}, s_{7}\right]$} & {$\left[s_{3}, s_{6}\right]$} & {$\left[s_{2}, s_{5}\right]$} \\
$A_{3}$ & {$\left[s_{4}, s_{7}\right]$} & {$\left[s_{6}, s_{7}\right]$} & {$\left[s_{2}, s_{5}\right]$} & {$\left[s_{5}, s_{8}\right]$} \\
$A_{4}$ & {$\left[s_{5}, s_{7}\right]$} & {$\left[s_{2}, s_{4}\right]$} & {$\left[s_{3}, s_{4}\right]$} & {$\left[s_{4}, s_{6}\right]$} \\
\hline
\end{tabular}

TABLE 3: The decision matrix $R_{3}$ given by $D_{3}$.

\begin{tabular}{lcccc}
\hline & $C_{1}$ & $C_{2}$ & $C_{3}$ & $C_{4}$ \\
\hline$A_{1}$ & {$\left[s_{4}, s_{6}\right]$} & {$\left[s_{3}, s_{5}\right]$} & {$\left[s_{4}, s_{6}\right]$} & {$\left[s_{1}, s_{4}\right]$} \\
$A_{2}$ & {$\left[s_{4}, s_{7}\right]$} & {$\left[s_{3}, s_{4}\right]$} & {$\left[s_{4}, s_{6}\right]$} & {$\left[s_{4}, s_{5}\right]$} \\
$A_{3}$ & {$\left[s_{7}, s_{7}\right]$} & {$\left[s_{4}, s_{5}\right]$} & {$\left[s_{3}, s_{5}\right]$} & {$\left[s_{6}, s_{6}\right]$} \\
$A_{4}$ & {$\left[s_{5}, s_{6}\right]$} & {$\left[s_{2}, s_{2}\right]$} & {$\left[s_{4}, s_{6}\right]$} & {$\left[s_{3}, s_{6}\right]$} \\
\hline
\end{tabular}

TABLE 4: The aggregated decision matrix $R$.

\begin{tabular}{ccccc}
\hline & $C_{1}$ & $C_{2}$ & $C_{3}$ & $C_{4}$ \\
\hline$A_{1}$ & {$\left[s_{3.412}, s_{4.876}\right]$} & {$\left[s_{1}, s_{2.987}\right]$} & {$\left[s_{1}, s_{3.540}\right]$} & {$\left[s_{1}, s_{4.094}\right]$} \\
$A_{2}$ & {$\left[s_{3.490}, s_{5.824}\right]$} & {$\left[s_{2.409}, s_{4.213}\right]$} & {$\left[s_{3.091}, s_{4.779}\right]$} & {$\left[s_{2.647}, s_{5.095}\right]$} \\
$A_{3}$ & {$\left[s_{5.132}, s_{7.104}\right]$} & {$\left[s_{3.521}, s_{4.551}\right]$} & {$\left[s_{2.364}, s_{5.099}\right]$} & {$\left[s_{4.680}, s_{5.587}\right]$} \\
$A_{4}$ & {$\left[s_{3.788}, s_{4.169}\right]$} & {$\left[s_{1}, s_{2.124}\right]$} & {$\left[s_{3.396}, s_{4.167}\right]$} & {$\left[s_{3.091}, s_{4.779}\right]$} \\
\hline
\end{tabular}

Step 1. The decision makers provide their evaluation values and construct the UL decision matrix $R_{k}=\left(r_{i j}^{k}\right)_{m \times n}(k=$ $1,2,3)$ as shown in Tables $1-3$, respectively.

Step 2. Suppose that $f(x)=8 /(x-1)$ and $g(x)=x^{2}$; utilize the EULPOWG operator to derive the aggregated decision matrix $R=\left(r_{i j}\right)_{4 \times 4}$, which is shown in Table 4 .

Taking $r_{11}$ as an example, we have $r_{11}=\left[s_{\alpha_{11}}, s_{\beta_{11}}\right]=$ $\operatorname{EULPOWG}\left(r_{11}^{1}, r_{11}^{2}, r_{11}^{3}\right)$, where $r_{11}^{1}=\left[s_{4}, s_{7}\right], r_{11}^{2}=\left[s_{3}, s_{4}\right]$, and $r_{11}^{3}=\left[s_{4}, s_{6}\right]$.

Then, by Definition 1, we can easily derive $r_{11}^{(1)}=r_{11}^{1}>$ $r_{11}^{(2)}=r_{11}^{3}>r_{11}^{(3)}=r_{11}^{2}$, where $r_{11}^{(i)}$ is the ith largest of $r_{11}^{j}$, $i=1,2,3$.

According to Definition 20, we have

$$
\begin{aligned}
T\left(\widetilde{s}_{(1)}\right) & =\sum_{i=2}^{3} \operatorname{Su}\left(\widetilde{s}_{(1)}, \widetilde{s}_{(i)}\right) \\
& =1-\frac{|4-4|+|7-6|}{2 \times 8}+1-\frac{|4-3|+|7-4|}{2 \times 8} \\
& =1.688
\end{aligned}
$$

$$
\begin{aligned}
T\left(\widetilde{s}_{(2)}\right) & =\sum_{\substack{i=1 \\
i \neq 2}}^{3} \operatorname{Su}\left(\widetilde{s}_{(2)}, \widetilde{s}_{(i)}\right) \\
& =1-\frac{|4-4|+|6-7|}{2 \times 8}+1-\frac{|4-3|+|6-4|}{2 \times 8} \\
& =1.75, \\
T\left(\widetilde{s}_{(3)}\right) & =\sum_{i=1}^{2} \operatorname{Su}\left(\widetilde{s}_{(3)}, \widetilde{s}_{(i)}\right) \\
& =1-\frac{|3-4|+|4-7|}{2 \times 8}+1-\frac{|4-3|+|6-4|}{2 \times 8} \\
& =1.563,
\end{aligned}
$$

and then,

$$
\begin{gathered}
R_{1}=V_{1}=1+T\left(\widetilde{s}_{(1)}\right)=2.668 \\
R_{2}=\sum_{j=1}^{2} V_{j}=\sum_{j=1}^{2}\left(1+T\left(\widetilde{s}_{(j)}\right)_{j}\right)=5.438 \\
T V=\sum_{j=1}^{3} V_{j}=\sum_{j=1}^{3}\left(1+T\left(\widetilde{s}_{(j)}\right)_{j}\right)=8.001 .
\end{gathered}
$$

Thus, we derive $u_{1}=0.111, u_{2}=0.351$, and $u_{3}=0.538$.

Consequently, by (40), we obtain

$$
\begin{aligned}
r_{11} & =\operatorname{EULPOWG}\left(r_{11}^{1}, r_{11}^{2}, r_{11}^{3}\right)=\prod_{i=1}^{n}\left(r_{11}^{(i)}\right)^{u_{i}} \\
& =\left[s_{4}, s_{7}\right]^{0.111} \otimes\left[s_{4}, s_{6}\right]^{0.351} \otimes\left[s_{3}, s_{4}\right]^{0.538} \\
& =\left[s_{3.412}, s_{4.876}\right] .
\end{aligned}
$$

Step 3. Aggregate $r_{i j}(j=1,2, \ldots, 4)$ to yield the overall performance values $r_{i}$ for each alternative $A_{i}(i=1,2, \ldots, 4)$ based on the EULWGM operator with the weight vector $w=$ $(0.3,0.1,0.2,0.4)$, as shown in Table 5 .

Step 4. By ranking $r_{i}(i=1,2,3,4)$ based on Definition 1 , the order of the alternatives can be obtained, which is listed in Table 5 . That is, $A_{3}$ is the best third-party logistics provider to be selected.

Just as Xu and Yager [22] pointed out, both the EULWPG and EULPOWG operators take the relationships between the arguments into account. The difference between such two operators is that the EULWPG operator stresses the importance of every ULV, while the EULPOWG operator emphasizes the importance of the ordered position of every ULV. In consequence, in the group decision making process, by using the EULWPG or EULPOWG operator, we can not only allow individual opinions to support each other in the aggregation process but also reduce the influence of excessive low or high evaluation values on the decision result by assigning them lower weights. 
TABLE 5: The collective overall preference values and the rankings of alternatives.

\begin{tabular}{lcccc}
\hline$A_{1}$ & $A_{2}$ & $A_{3}$ & $A_{4}$ & Ranking \\
\hline$\left[s_{1}, s_{4.045}\right]$ & {$\left[s_{2.925}, s_{5.131}\right]$} & {$\left[s_{4.008}, s_{5.763}\right]$} & {$\left[s_{1}, s_{4.065}\right]$} & $A_{3}>A_{2}>A_{4}>A_{1}$ \\
\hline
\end{tabular}

\section{Conclusions}

In this paper, the MAGDM problems with UL information are investigated. Motivated by the ideal of Lan et al. [18], the extended triangular norm is defined, based on which several UL geometric aggregation operators are proposed, such as the EULGM operator, EULWGM operator, EULOGW operator, EULPG operator, EULWPG operator, and EULPOWG operator, together with their properties. In the process of decision making, these UL aggregation operators can avoid the limitations of existing linguistic aggregation operators; that is, the results derived in the computing processes sometimes may be counterintuitive or beyond the discourse domain of the linguistic variable. As a result, more intuitive and acceptable results may be obtained by these aggregation operators proposed.

\section{Conflict of Interests}

The author declares that there is no conflict of interests regarding the publication of this paper.

\section{Acknowledgments}

This work was supported by the National Natural Science Foundation of China under Grant no. U1304701, the HighLevel Talent Project of Henan University of Technology under Grant no. 2013BS015, and the Plan of Nature Science Fundamental Research in Henan University of Technology under Grant no. 2013JCYJ14.

\section{References}

[1] M. Delgado, J. L. Verdegay, and M. A. Vila, "On aggregation operations of linguistic labels," International Journal of Intelligent Systems, vol. 8, no. 3, pp. 351-370, 1993.

[2] Y. Dong, Y. Xu, H. Li, and B. Feng, "The OWA-based consensus operator under linguistic representation models using position indexes," European Journal of Operational Research, vol. 203, no. 2, pp. 455-463, 2010.

[3] L. Godo and V. Torra, "On aggregation operators for ordinal qualitative information," IEEE Transactions on Fuzzy Systems, vol. 8, no. 2, pp. 143-154, 2000.

[4] F. Herrera, E. Herrera-Viedma, and J. L. Verdegay, "A model of consensus in group decision making under linguistic assessments," Fuzzy Sets and Systems, vol. 78, no. 1, pp. 73-87, 1996.

[5] F. Herrera and E. Herrera-Viedma, "Aggregation operators for linguistic weighted information," IEEE Transactions on Systems, Man, and Cybernetics Part A: Systems and Humans., vol. 27, no. 5, pp. 646-656, 1997.

[6] F. Herrera and L. Martínez, "A 2-tuple fuzzy linguistic representation model for computing with words," IEEE Transactions on Fuzzy Systems, vol. 8, no. 6, pp. 746-752, 2000.
[7] F. Herrera, E. Herrera-Viedma, and J. L. Verdegay, "A sequential selection process in group decision making with a linguistic assessment approach," Information Sciences, vol. 85, no. 4, pp. 223-239, 1995.

[8] F. Herrera and E. Herrera-Viedma, "Linguistic decision analysis: steps for solving decision problems under linguistic information," Fuzzy Sets and Systems, vol. 115, no. 1, pp. 67-82, 2000.

[9] K. K. F. Yuen and H. C. W. Lau, "A Linguistic PossibilityProbability Aggregation Model for decision analysis with imperfect knowledge," Applied Soft Computing Journal, vol. 9, no. 2, pp. 575-589, 2009.

[10] C. Porcel and E. Herrera-Viedma, "Dealing with incomplete information in a fuzzy linguistic recommender system to disseminate information in university digital libraries," KnowledgeBased Systems, vol. 23, no. 1, pp. 32-39, 2010.

[11] F. J. Cabrerizo, I. J. Pérez, and E. Herrera-Viedma, "Managing the consensus in group decision making in an unbalanced fuzzy linguistic context with incomplete information," KnowledgeBased Systems, vol. 23, no. 2, pp. 169-181, 2010.

[12] Z. S. Xu, "Deviation measures of linguistic preference relations in group decision making," Omega, vol. 33, no. 3, pp. 249-254, 2005.

[13] Z. S. Xu, "Uncertain linguistic aggregation operators based approach to multiple attribute group decision making under uncertain linguistic environment," Information Sciences, vol. 168, no. 1-4, pp. 171-184, 2004.

[14] Z. S. Xu, "Induced uncertain linguistic OWA operators applied to group decision making," Information Fusion, vol. 7, no. 2, pp. 231-238, 2006.

[15] Z. S. Xu, "An approach based on the uncertain LOWG and induced uncertain LOWG operators to group decision making with uncertain multiplicative linguistic preference relations," Decision Support Systems, vol. 41, no. 2, pp. 488-499, 2006.

[16] G.-W. Wei, "Uncertain linguistic hybrid geometric mean operator and its application to group decision making under uncertain linguistic environment," International Journal of Uncertainty, Fuzziness and Knowlege-Based Systems, vol. 17, no. 2, pp. 251-267, 2009.

[17] B. Peng, C. Ye, and S. Zeng, "Uncertain pure linguistic hybrid harmonic averaging operator and generalized interval aggregation operator based approach to group decision making," Knowledge-Based Systems, vol. 36, pp. 175-181, 2012.

[18] J. B. Lan, Q. Sun, Q. M. Chen, and Z. X. Wang, "Group decision making based on induced uncertain linguistic OWA operators," Decision Support Systems, vol. 55, no. 1, pp. 296-303, 2013.

[19] G. W. Wei, X. F. Zhao, R. Lin, and H. J. Wang, "Uncertain linguistic Bonferroni mean operators and their application to multiple attribute decision making," Applied Mathematical Modelling, vol. 37, no. 7, pp. 5277-5285, 2013.

[20] D.-H. Peng, T.-D. Wang, C.-Y. Gao, and H. Wang, "Multigranular uncertain linguistic prioritized aggregation operators and their application to multiple criteria group decision making," Journal of Applied Mathematics, vol. 2013, Article ID 857916, 13 pages, 2013. 
[21] R. R. Yager, “The power average operator," IEEE Transactions on Systems, Man, and Cybernetics Part A:Systems and Humans., vol. 31, no. 6, pp. 724-731, 2001.

[22] Z. Xu and R. R. Yager, "Power-geometric operators and their use in group decision making," IEEE Transactions on Fuzzy Systems, vol. 18, no. 1, pp. 94-105, 2010.

[23] L. Zhou and H. Chen, "A generalization of the power aggregation operators for linguistic environment and its application in group decision making," Knowledge-Based Systems, vol. 26, pp. 216-224, 2012.

[24] Y. J. Xu and H. M. Wang, "Approaches based on 2-tuple linguistic power aggregation operators for multiple attribute group decision making under linguistic environment," Applied Soft Computing Journal, vol. 11, no. 5, pp. 3988-3997, 2011.

[25] Y. J. Xu, J. M. Merigó, and H. M. Wang, "Linguistic power aggregation operators and their application to multiple attribute group decision making," Applied Mathematical Modelling, vol. 36, no. 11, pp. 5427-5444, 2012.

[26] Z. Xu, "A method based on linguistic aggregation operators for group decision making with linguistic preference relations," Information Sciences, vol. 166, no. 1-4, pp. 19-30, 2004.

[27] Z. S. Xu, "Algorithm for priority of fuzzy complementary judgment matrix," Journal of Systems Engineering, vol. 16, pp. 311-314, 2001.

[28] G. Klir and B. Yuan, Fuzzy Sets and Fuzzy Logic: Theory and Applications, Prentice Hall, Upper Saddle River, NJ, USA, 1995.

[29] E. P. Klement, R. Mesiar, and E. Pap, Triangular Norms, Kluwer Academic Publishers, Dordrecht, The Netherlands, 2000. 


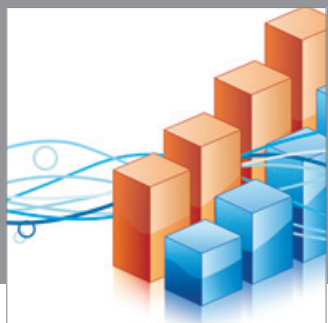

Advances in

Operations Research

mansans

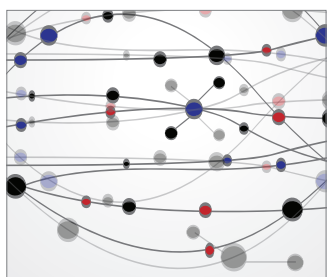

The Scientific World Journal
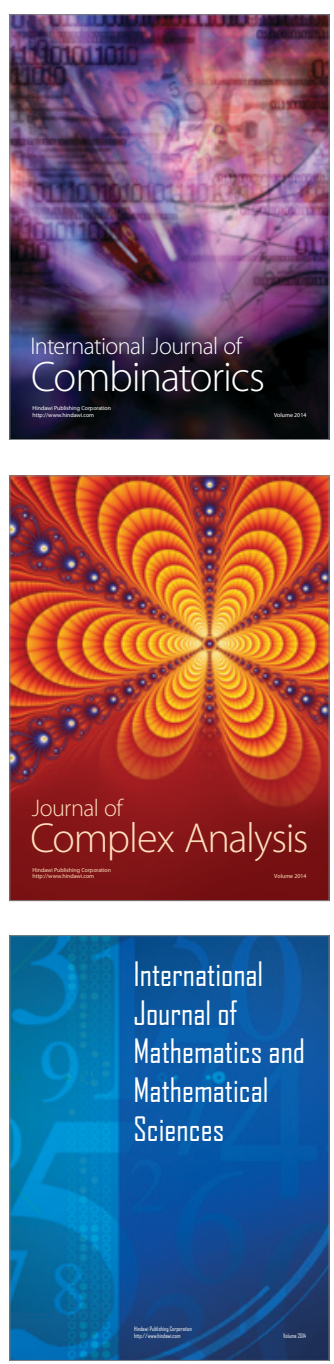
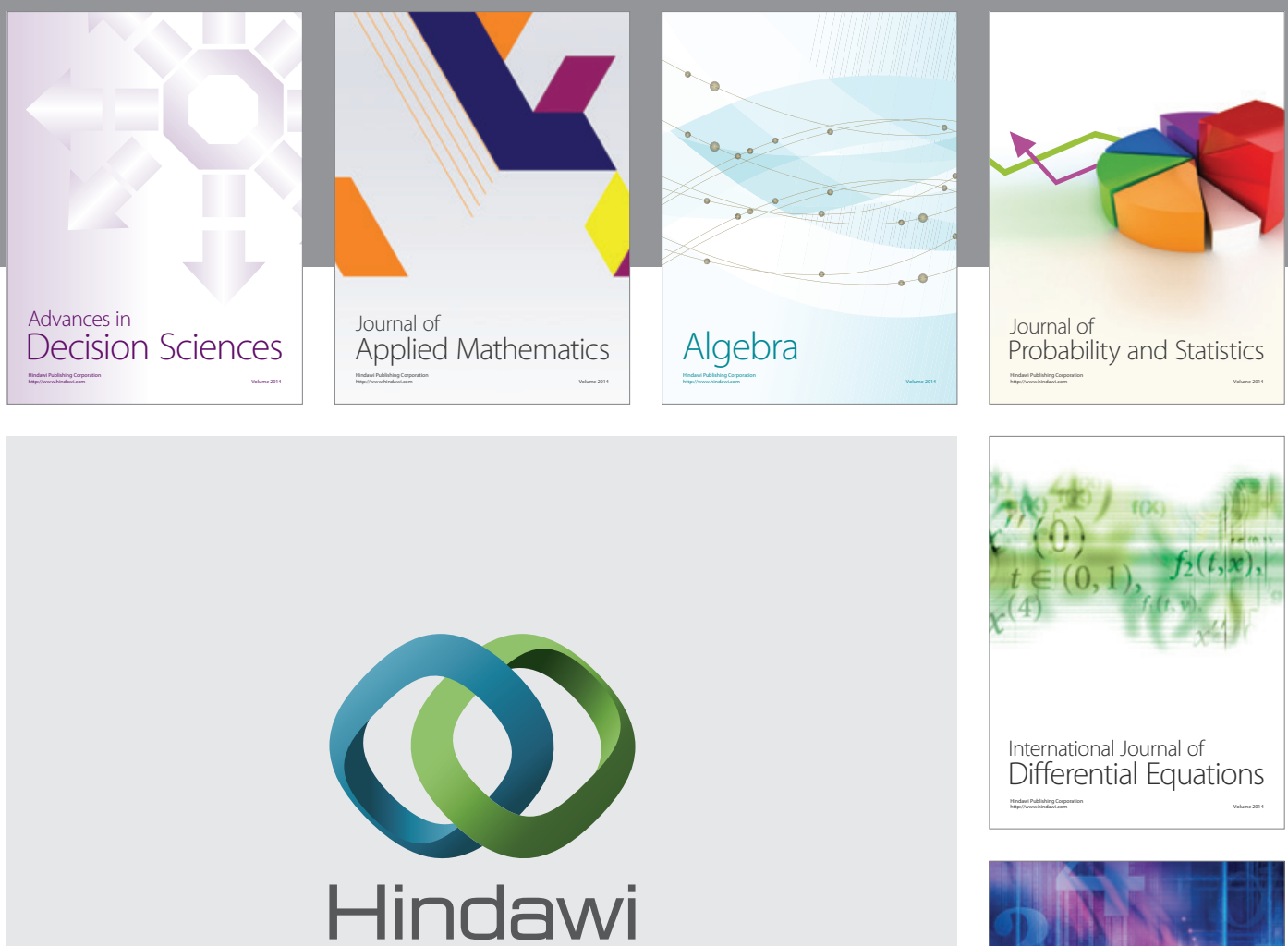

Submit your manuscripts at http://www.hindawi.com
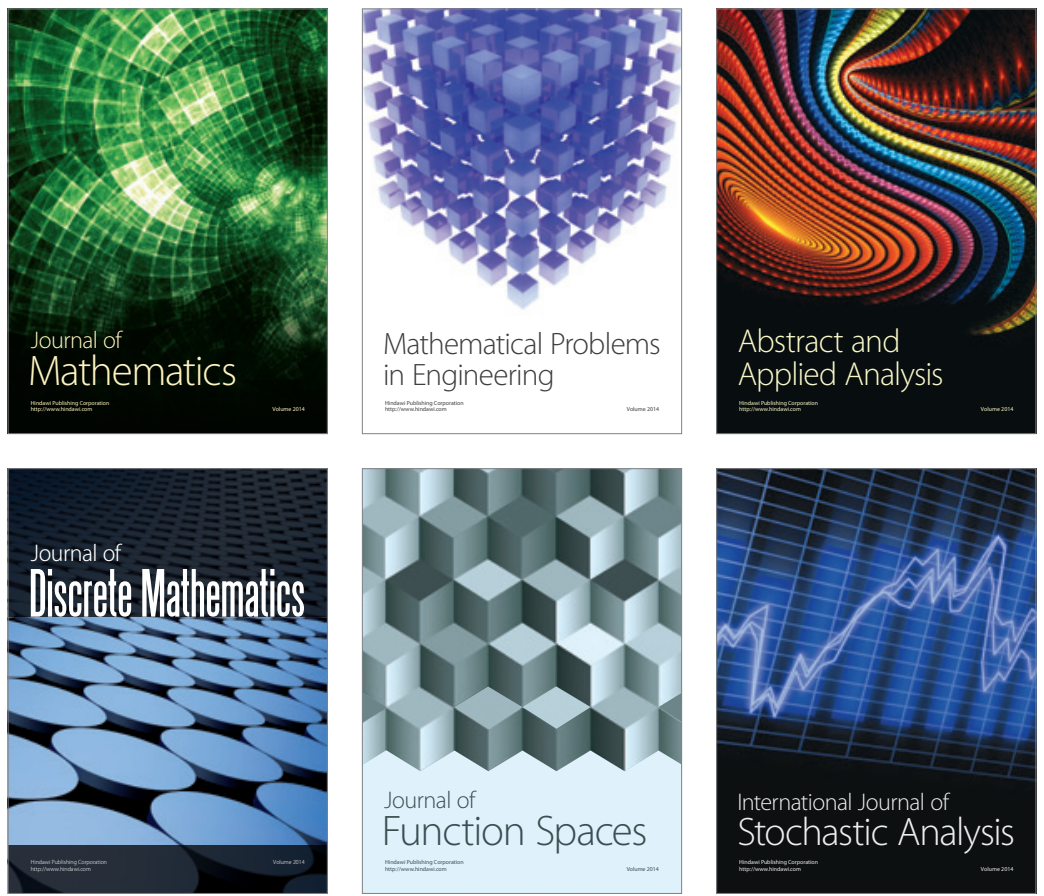

Journal of

Function Spaces

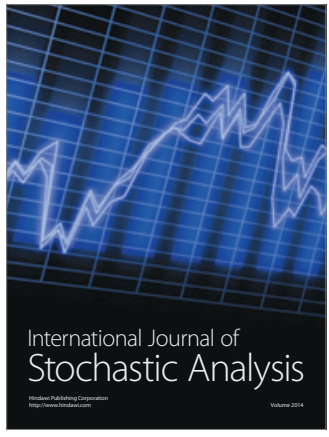

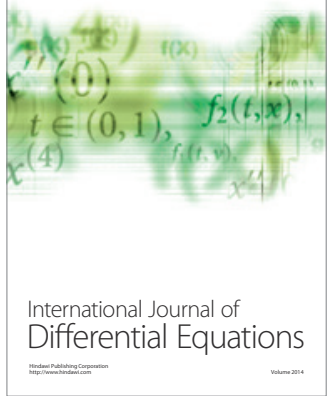
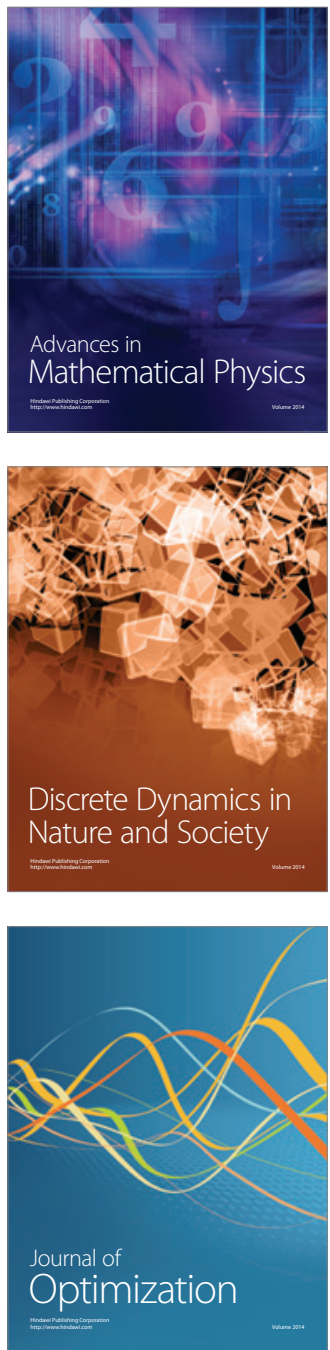